\title{
Uncertainty propagation by using spectral methods: a practical application to a two-dimensional turbulence fluid model
}

\author{
Fabio Riva, ${ }^{1, *}$ Lucio Milanese, ${ }^{2}$ and Paolo Ricci ${ }^{1}$ \\ ${ }^{1}$ École Polytechnique Fédérale de Lausanne (EPFL), \\ Swiss Plasma Center (SPC), CH-1015 Lausanne, Switzerland \\ ${ }^{2}$ Plasma Science and Fusion Center, \\ Massachusetts Institute of Technology, \\ Cambridge, Massachusetts 02139, USA
}

\begin{abstract}
To reduce the computational cost of the uncertainty propagation analysis, which is used to study the impact of input parameter variations on the results of a simulation, a general and simple to apply methodology based on decomposing the solution to the model equations in terms of Chebyshev polynomials is discussed. This methodology, based on the work by Scheffel [American Journal of Computational Mathematics 02, 173-193 (2012)], approximates the model equation solution with a semi-analytic expression that depends explicitly on time, spatial coordinates, and input parameters. By employing a weighted residual method, a set of nonlinear algebraic equations for the coefficients appearing in the Chebyshev decomposition is then obtained. The methodology is applied to a two-dimensional Braginskii model used to simulate plasma turbulence in basic plasma physics experiments and in the scrape-off layer of tokamaks, in order to study the impact on the simulation results of the input parameter that describes the parallel losses. The uncertainty that characterizes the time-averaged density gradient lengths, time-averaged densities, and fluctuation density level are evaluated. A reasonable estimate of the uncertainty of these distributions can be obtained with a single reduced-cost simulation.
\end{abstract}

\footnotetext{
*Electronic address: fabio.riva@epfl.ch
} 


\section{INTRODUCTION}

In order to assess the reliability of a simulation and validate it against experimental measurements, it is essential to estimate the uncertainties affecting its numerical results [1-3]. These uncertainties stem from numerically solving the model equations with finite precision, and from the use of input parameters that are not precisely known or accurately measured. While a rigorous methodology for estimating the numerical errors affecting plasma turbulence simulations has been recently proposed (see, e.g., Refs. $[4,5]$ ), the absence of a rigorous procedure to investigate uncertainty propagation through a plasma turbulence model persists and motivates the work illustrated in the present paper.

An analytical study of uncertainty propagation is unfeasible for complex physical models such as the ones describing plasma turbulence. In these cases uncertainty propagation is approached numerically. The most straightforward strategy to study uncertainty propagation is based on the assumption that the uncertainty on an input parameter is described by a probability distribution [6]. A sample of input parameters is then randomly generated according to such a distribution and a simulation is performed for each input of the sample. A distribution of simulation results it thus obtained. From this distribution it is possible to evaluate the uncertainty affecting the point-by-point solution values or solution functionals. While conceptually simple, this approach is usually not applicable to plasma turbulence simulations because of the high computational cost and of the large number of input parameters typically involved. Despite the fact that sophisticated procedures have been developed to predict the response of the model to variation of input parameters using the smallest possible number of simulations, such as Bayesian analysis [6], multifidelity Monte-Carlo estimations [7], and the stochastic response surface methodology [8, 9], to our knowledge they have never been employed by the plasma physics community. Rather, uncertainty propagation is typically investigated in plasma physics by performing sensitivity scans (see, e.g., Ref. [10]). More precisely, the input parameters $x_{i}$, which the model is more sensitive to, are identified. Then, one simulation is performed by using all input parameters at their reference values $x_{i}=\bar{x}_{i}$, for all $i$. In addition, two more simulations are run for each identified input parameter, using $x_{i}=\bar{x}_{i}$ for all $i \neq j$ and $x_{j}=\bar{x}_{j} \pm \epsilon_{x_{j}}$, with $\epsilon_{x_{j}}$ the uncertainty on the reference value $\bar{x}_{j}$. Finally, the uncertainty is identified by assuming a linear dependence of the simulation results on the input parameters. While computationally less demanding 
than a simple statistical analysis, this approach is still considerably expensive, particularly for plasma turbulence simulations involving a large number of input parameters. Moreover, the solution of differential equations practically never depends linearly on the input parameters [6]. As a consequence, the numerical results of the simulation with $x_{i}=\bar{x}_{i}$ generally differ from averaging the simulation results obtained by performing a number of simulations for randomly distributed $x_{i}$. A more rigorous approach for analyzing uncertainty propagation is therefore necessary. In particular, developing a methodology allowing for low-cost, low-resolution simulations can be very helpful.

To estimate the dependence of the code results on the input parameters, in the present paper we consider a methodology, based on the work presented by Scheffel in Ref. [11], which consists in a decomposition of the solution to the model equations in terms of Chebyshev polynomials along the time, spatial, and input parameter coordinates. More precisely, a series of Chebyshev polynomials is used to represent the solution of a differential equation and to express its dependence on the temporal, spatial, and input variables. A weighted residual method (WRM) is then employed to deduce a set of algebraic equations, thus making it possible to numerically evaluate the coefficients appearing in the Chebyshev decomposition and obtaining a semi-analytical expression for the solution with explicit dependence on the input parameters. This allows us to determine the parametric dependence of the solution, while avoiding to perform a set of simulations for different input values, and to investigate the impact of the input parameters on the model solution by performing a single simulation. We apply the proposed methodology to a two-dimensional fluid model used to investigate the plasma dynamics in basic plasma physics experiments, such as linear devices [12] and simple magnetized torus (SMT) [13-15], and in the tokamak scrape-off layer (SOL) [16, 17]. The model is based on the drift-reduced Braginskii equations and evolves in time the plasma density, the plasma potential, and the electron temperature. The algebraic system of equations resulting from projecting the model equations on Chebyshev's space is implemented in a simulation code. We then use the simulation results to assess the influence of uncertainties affecting the input parameter that describes the parallel losses on the density profile.

We note that the use of fully spectral methods to solve differential equations is far from new, as they have been widely employed by the computational fluid dynamics community (see, e.g., Ref. [18]). However, fully spectral codes were rarely used to investigate plasma physics problems and, to our knowledge, their use remained limited to the study of plasma 
flows and linear stability analysis (see, e.g., Refs. [11, 19]), not being applied to the analysis of uncertainty propagation in nonlinear plasma turbulence simulations. This motivates the study illustrated in the present paper.

This paper is structured as follows. After the Introduction, in Sec. II we present the spectral method we employ to solve a set of partial differential equations. More precisely, we discuss the approximation of the model equation solution with Chebyshev polynomials and the application of the WRM. Then, in Sec. III we illustrate the two-dimensional drift-reduced Braginskii model we consider in the present paper. In Sec. IV we apply the Chebyshev spectral method to study uncertainty propagation through the drift-reduced Braginskii model. The Conclusions follow. The approximation of differential and nonlinear operators, the treatment of the initial condition, and the application of the boundary conditions in the Chebyshev spectral domain are the subject of Appendixes A, B, and C, respectively.

\section{CHEBYSHEV SPECTRAL METHOD}

In this section we discuss the application of the WRM to obtain an approximated solution of a differential equation in the Chebyshev spectral space. We note that the methodology illustrated in the rest of the present section is based on the work presented in Ref. [11]. We consider an initial value parabolic or hyperbolic partial differential equation

$$
\frac{\partial \mathbf{u}}{\partial t}=D_{\mathbf{p}}\{\mathbf{u}\}+\mathbf{S}
$$

with the exact solution $\mathbf{u}=\mathbf{u}(t, \mathbf{x} ; \mathbf{p})$ that depends on time $t \in\left[t_{0}, t_{1}\right]$, space coordinates $\mathbf{x} \in\left[\mathbf{x}_{0}, \mathbf{x}_{1}\right]$, and a set of parameters $\mathbf{p} \in\left[\mathbf{p}_{0}, \mathbf{p}_{1}\right]$ (the indexes 0 and 1 referring to the lower and upper boundaries of the considered domains). Here $D_{\mathbf{p}}$ is a linear or nonlinear differential operator depending on $\mathbf{p}$ and acting on $\mathbf{u}$, and $\mathbf{S}=\mathbf{S}(t, \mathbf{x} ; \mathbf{p})$ a given source term. Equation (1) is completed by an initial condition $\mathbf{u}\left(t_{0}, \mathbf{x} ; \mathbf{p}\right)=\mathbf{u}_{0}(\mathbf{x} ; \mathbf{p})$, with $\mathbf{u}_{0}$ a given function, and a set of boundary conditions for the spatial domain. For simplicity, in

the following of this section we consider a one dimensional function $u=u(t, x ; p)$ depending on one spatial dimension $x$ and one input parameter $p$. The generalization to equations with more dimensions and parameters does not present conceptual difficulties.

The WRM is used to solve differential equations by approximating $u$ with a linear combination of independent basis functions. We choose the Chebyshev polynomials of the first 
kind $T_{n}(x)=\cos \left(n \cos ^{-1} x\right)$, defined for $x \in[-1,1]$, as basis functions. Hereafter $T_{n}$ are simply named Chebyshev polynomials. We note that $n \in \mathbb{N}$ constitutes the degree of the Chebyshev polynomial, that Chebyshev polynomials are orthogonal over the weight function $w(x)=\left(1-x^{2}\right)^{-1}$ in the interval $x \in[-1,1]$, i.e.

$$
\int_{-1}^{1} \frac{T_{m}(x) T_{n}(x)}{\sqrt{1-x^{2}}} d x=\frac{\pi}{2}\left(\delta_{m n}+\delta_{m 0} \delta_{n 0}\right)
$$

where $\delta_{m n}$ is the Kronecker delta $\left(\delta_{m n}=1\right.$ if $m=n$ and $\delta_{m n}=0$ otherwise), and that they are characterized by the minimax property, i.e. the expansion of a continuous function $f(x) \simeq \sum_{l=0}^{L}{ }^{\prime} a_{l} T_{l}(\chi)$, with

$$
a_{l}=\frac{2}{\pi B_{x}} \int_{x_{0}}^{x_{1}} f(x) \frac{T_{l}(\chi)}{\sqrt{1-\chi^{2}}} d x
$$

provides the most accurate approximation of $f$ under the maximum norm, $\|-\|_{\infty}$, for a polynomial of degree $L$ [20]. The minimax property motivates the choice of using the $T_{n}$ polynomials as basis functions, since it implies that the best approximation of $f$ at order $L$ in Chebyshev space is simply the series truncated at $l=L$. Consequently, we approximate

$$
u(t, x ; p) \simeq \hat{u}(t, x ; p)=\sum_{k=0}^{K}{ }^{\prime} \sum_{l=0}^{L}, \sum_{m=0}^{M}{ }^{\prime} a_{k l m} T_{k}(\tau) T_{l}(\chi) T_{m}(\sigma),
$$

where $a_{l k m}$ are constant coefficients, $K+1, L+1, M+1$ the numbers of Chebyshev polynomials used for the expansion in time, real space, and input parameter space, respectively, and

$$
\tau=\frac{t-A_{t}}{B_{t}}, \chi=\frac{x-A_{x}}{B_{x}}, \sigma=\frac{p-A_{p}}{B_{p}},
$$

with $A_{t}=\left(t_{1}+t_{0}\right) / 2$ and $B_{t}=\left(t_{1}-t_{0}\right) / 2$ (similar definitions apply to the other quantities), such that $\tau, \chi, \sigma \in[-1,1]$. Primes on summation signs indicate that the 0 -th term of each sum is multiplied by a factor $1 / 2$, i.e.

$$
\sum_{n=0}^{N}{ }^{\prime} a_{n} T_{n}(\chi)=\frac{a_{0}}{2} T_{0}(\chi)+a_{1} T_{1}(\chi)+a_{2} T_{2}(\chi)+\ldots+a_{N} T_{N}(\chi) .
$$

Integrating Eq. (1) in time, i.e. writing

$$
u(t, x ; p)=u_{0}(x ; p)+\int_{t_{0}}^{t}\left(D_{p}\{u\}+S\right) d t^{\prime},
$$

we define the residual $R$ as

$$
R(t, x ; p)=\hat{u}(t, x ; p)-\left[u_{0}(x ; p)+\int_{t_{0}}^{t} d t^{\prime} D_{p}\left\{\hat{u}\left(t^{\prime}, x ; p\right)\right\}+S\left(t^{\prime}, x ; p\right)\right] .
$$


In order to estimate the coefficients $a_{l k m}$, the equation

$$
\int_{t_{0}}^{t_{1}} \int_{x_{0}}^{x_{1}} \int_{p_{0}}^{p_{1}} R(t, x ; p) W_{k l m}(t, x ; p) d t d x d p=0
$$

is solved for all $0 \leq k \leq K, 0 \leq l \leq L, 0 \leq m \leq M$, with $W_{k l m}(t, x ; p)$ a set of ( $K+$ 1) $(L+1)(M+1)$ properly chosen weight functions. To take advantage of the orthogonality property of Chebyshev polynomials, we choose

$$
W_{k l m}(t, x ; p)=\frac{T_{k}(\tau)}{\sqrt{1-\tau^{2}}} \frac{T_{l}(\chi)}{\sqrt{1-\chi^{2}}} \frac{T_{m}(\sigma)}{\sqrt{1-\sigma^{2}}} .
$$

We now express Eq. (8) in a form useful for further progress. Using the orthogonality property, Eq. (2), we have

$$
\int_{t_{0}}^{t_{1}} \int_{x_{0}}^{x_{1}} \int_{p_{0}}^{p_{1}} \hat{u}(t, x ; p) W_{k l m}(t, x ; p) d t d x d p=B_{t} B_{x} B_{p}\left(\frac{\pi}{2}\right)^{3} a_{k l m} .
$$

Moreover, we approximate

$$
\int_{t_{0}}^{t} S\left(t^{\prime}, x ; p\right) d t^{\prime} \simeq \sum_{k=0}^{K}{ }^{\prime} \sum_{l=0}^{L}, \sum_{m=0}^{M}{ }^{\prime} S_{k l m} T_{k}(\tau) T_{l}(\chi) T_{m}(\sigma)
$$

and

$$
u_{0}(x ; p) \simeq \sum_{l=0}^{L}, \sum_{m=0}^{M}{ }^{\prime} b_{l m} T_{l}(\chi) T_{m}(\sigma)
$$

Finally, we write

$$
\int_{t_{0}}^{t} D_{p}\left\{\hat{u}\left(t^{\prime}, x ; p\right)\right\} d t^{\prime} \simeq \sum_{k=0}^{K}{ }^{\prime} \sum_{l=0}^{L}{ }^{\prime} \sum_{m=0}^{M}{ }^{\prime} A_{k l m} T_{k}(\tau) T_{l}(\chi) T_{m}(\sigma),
$$

where $A_{k l m}$ are assumed to be known functions of the $a_{k l m}$ coefficients. Using again the orthogonality property of Chebyshev polynomials, Eq. (9) yields

$$
a_{k l m}=2 \delta_{k 0} b_{l m}+A_{k l m}+S_{k l m}
$$

which is a set of $(K+1)(L+1)(M+1)$ coupled algebraic equations. Equation (15) approximates Eq. (7) in Chebyshev space. The term $2 \delta_{k 0} b_{l m}$ represents the initial condition, and $A_{k l m}$ and $S_{k l m}$ represent the projection along the Chebyshev basis of the operator $D_{p}$ applied to $u$ and of the source term, respectively.

In order to solve Eq. (15) for $a_{k l m}$, one has to deduce first an explicit relation between $A_{k l m}$ and the Chebyshev expansion of $D_{p}\{\hat{u}\}$. Approximating

$$
D_{p}\{\hat{u}\} \simeq \sum_{k=0}^{K}{ }^{\prime} \sum_{l=0}^{L}, \sum_{m=0}^{M}{ }^{\prime} c_{k l m} T_{k}(\tau) T_{l}(\chi) T_{m}(\sigma)
$$


we have

$$
\begin{array}{rlrl}
A_{k l m}=\frac{B_{t}}{2 k}\left(c_{k-1, l, m}-c_{k+1, l, m}\right) & 0<k \leq K-1 \\
A_{k l m}=\frac{B_{t}}{2 k} c_{k-1, l, m} & k=K .
\end{array}
$$

Since

$$
\int_{t_{0}}^{t_{0}} D_{p}\left\{\hat{u}\left(t^{\prime}, x ; p\right)\right\} d t^{\prime}=0
$$

the coefficients $A_{0 l m}$ are found by imposing

$$
A_{0 l m}=-2 \sum_{k=1}^{K} A_{k l m}(-1)^{k} .
$$

Finally, in order to solve Eqs. (15) for the coefficients $a_{k l m}$, it is necessary to express the operator $D_{p}$ in the Chebyshev spectral domain and determine the coefficients $c_{k l m}$ as a function of $a_{k l m}$, as detailed in Appendix A, and to apply the initial and boundary conditions in Chebyshev space, as discussed in Appendixes B and C, respectively.

To conclude our discussion on the use of the WRM with Chebyshev polynomials, we would like to make two remarks. First, while Eq. (7) constitutes a single partial differential equation, Eq. (15) represents a set of coupled algebraic equations that can be solved, either analytically or numerically, to compute the coefficients $a_{k l m}$ and obtain an approximated semi-analytical solution of Eq. (7) with explicit dependence on $p$. Second, in general, Eq. (15) cannot be solved analytically if $D_{p}$ is a nonlinear operator. In such a case, Eq. (15) must be solved numerically, usually employing an iterative solver. Since the latter might need a good initial guess to converge, it is suitable to decompose the time interval into sub-domains [21]. More precisely, the time coordinate $t \in\left[t_{0}, t_{1}\right]$ is decomposed in $N$ subdomains of length $\Delta t^{i}>0$, with $i=1, \ldots, N$ and $t_{1}-t_{0}=\sum_{i=1}^{N} \Delta t^{i}$. Equation (15) is then solved for $t \in\left[t_{0}, t_{0}+\Delta t^{1}\right]$ using the initial guess $a_{k l m}^{1}=2 \delta_{0 k} b_{l m}^{1}$ ( $a_{k l m}^{i}$ and $b_{l m}^{i}$ denote respectively the coefficients $a_{k l m}$ and $b_{l m}$ in the sub-domain $\left.\left[t_{0}+\sum_{j=1}^{i-1} \Delta t^{j}, t_{0}+\sum_{j=1}^{i} \Delta t^{j}\right]\right)$. Finally, for the subsequent sub-domains we impose $b_{l m}^{i+1}=\sum_{k=0}^{K}{ }^{\prime} a_{k l m}^{i}$ and we use the initial guess $a_{k l m}^{i+1}=a_{k l m}^{i}$ for the solution of Eq. (15) in the sub-domain $i+1$.

\section{TWO-DIMENSIONAL DRIFT-REDUCED BRAGINKSII EQUATIONS}

To illustrate an application of the Chebyshev spectral method to the study of uncertainty propagation, we consider here a two-dimensional drift-reduced Braginskii model, used in the 
past to simulate plasma turbulence in linear and SMT devices (see Ref. [10] for a detailed discussion of this model). We focus here on the SMT configuration, where the magnetic field is obtained by superimposing a toroidal magnetic field on a vertical magnetic field. This results in helical field lines that wind around the toroidal vacuum vessel from the bottom to the top of the device. The SMT configuration, implemented in a number of basic plasma physics experiments such as TORPEX [22, 23] and Texas Helimak [24], is of interest to the plasma physics community because it offers a simple and well diagnosed scenario to study the turbulent transport resulting from instabilities such as interchange modes and drift waves, which are present also in the tokamak SOL. In fact, models similar the the one used to investigate the SMT plasma dynamics have been used also for the tokamak SOL (see, e.g., Refs. [16, 17]).

To describe the SMT configuration, we indicate with $x$ the radial coordinate, with $z$ the coordinate along the magnetic field (approximately the toroidal direction), and with $y$ the coordinate perpendicular to $x$ and $z$ (approximately the vertical direction). Because of the high collisionality typical of SMT experiments, it is justified to model the plasma dynamics with a set of drift-reduced Braginskii equations. Focusing on the interchange-like turbulent regime observed for a sufficiently high magnetic field [15], for which $k_{z} \simeq 0$, and under the assumption of cold ions, that allows neglecting the ion temperature dynamics, it is possible to integrate the drift-reduced Braginskii equations discussed in Ref. [25] in the parallel direction, in order to evolve the line-averaged density $n(y, x)=\int n(y, x, z) d z / L_{\|}$, electrostatic potential $\phi(y, x)=\int \phi(y, x, z) d z / L_{\|}$, and electron temperature $T_{e}(y, x)=\int T_{e}(y, x, z) d z / L_{\|}$, with $L_{\|} \simeq 2 \pi N_{\text {turns }} R_{0}$ the magnetic field line length, $R_{0}$ the SMT major radius, and $N_{\text {turns }}$ the number of turns of the magnetic field line in the device. Neglecting the parallel electron thermal conductivity term (according to $k_{z} \simeq 0$ ), using the Boussinesq approximation [2628] to simplify the vorticity equation, assuming an infinite aspect ratio, and applying Bohm's boundary conditions at the sheath edge, $v_{\| i}= \pm \sqrt{T_{e}}$ and $v_{\| e}= \pm \sqrt{T_{e}} \exp \left(\Lambda-\phi / T_{e}\right)$, the 
resulting system of equations is

$$
\begin{aligned}
\frac{\partial n}{\partial t}= & -R_{0}\{\phi, n\}+2\left(\frac{\partial n}{\partial y}-n \frac{\partial \phi}{\partial y}\right)-\sigma n \sqrt{T_{e}} \exp \left(\Lambda-\frac{\phi}{T_{e}}\right)+\mathcal{D}_{n}(n)+S_{n}, \\
\frac{\partial \omega}{\partial t}= & -R_{0}\{\phi, \omega\}+\frac{2}{n} \frac{\partial p_{e}}{\partial y}+\sigma \sqrt{T_{e}}\left[1-\exp \left(\Lambda-\frac{\phi}{T_{e}}\right)\right]+\mathcal{D}_{\omega}(\omega), \\
\frac{\partial T_{e}}{\partial t}= & -R_{0}\left\{\phi, T_{e}\right\}+\frac{4 T_{e}}{3}\left(\frac{1}{n} \frac{\partial p_{e}}{\partial y}+\frac{5}{2} \frac{\partial T_{e}}{\partial y}-\frac{\partial \phi}{\partial y}\right) \\
& -\frac{2 \sigma T_{e} \sqrt{T_{e}}}{3}\left[1.71 \exp \left(\Lambda-\frac{\phi}{T_{e}}\right)-0.71\right]+\mathcal{D}_{T_{e}}\left(T_{e}\right)+S_{T_{e}},
\end{aligned}
$$

where $\Lambda \simeq 3$ for hydrogen plasmas, $\omega=\nabla_{\perp}^{2} \phi, \nabla_{\perp}^{2} A=\partial_{x}^{2} A+\partial_{y}^{2} A$ and $\{\phi, A\}=$ $\partial_{y} \phi \partial_{x} A-\partial_{x} \phi \partial_{y} A$, being $A=n, \phi, \omega, T_{e}$. Here the source terms $S_{n}$ and $S_{T_{e}}$ are used to mimic the density and temperatures sources, e.g., due to a resonance at the electron cyclotron frequency, and they are assumed vertically constant, with a Gaussian shape in the radial direction. Small perpendicular diffusion terms of the form $\mathcal{D}_{A}(A)=D_{A} \nabla_{\perp}^{2} A$, with $D_{A}$ a constant coefficient, are added for the numerical solution of the equations, with a negligible impact on the results. The quantities appearing in Eqs. (20)-(22) and in the rest of the present paper are normalized according to (tilde denotes a physical quantity in SI units): $t=\tilde{t} /\left(\tilde{R} / \tilde{c}_{s 0}\right), n=\tilde{n} / \tilde{n}_{0}, T_{e}=\tilde{T}_{e} / \tilde{T}_{e 0}, \phi=e \tilde{\phi} / \tilde{T}_{e 0}, R_{0}=\tilde{R} / \tilde{\rho}_{s 0}$, where $\tilde{n}_{0}$ and $\tilde{T}_{e 0}$ are reference density and electron temperature, $\tilde{R}$ is the SMT major radius, and $\tilde{c}_{s 0}$ and $\tilde{\rho}_{s 0}$ are given by $\tilde{c}_{s 0}=\sqrt{\tilde{T}_{e 0} / m_{i}}$ and $\tilde{\rho}_{s 0}=\tilde{c}_{s 0} m_{i} /\left(e \tilde{B}_{0}\right)$, being $\tilde{B}_{0}$ the magnetic field amplitude on axis. Distances are normalized to $\tilde{\rho}_{s 0}$.

The parameter $\sigma$ in Eqs. (20)-(22) is used to model the parallel losses at the magnetic pre-sheath (MP) entrance. Approaching the vessel wall, a decrease of $\phi$ is expected [29], which results in the ion acceleration to the ion sound speed. This mechanism leads to a decrease of $n$ at the MP entrance, $n_{M P}$, with respect to the density observed far away from the sheath. The simplest model, which assumes being in a sheath-limited regime with isothermal electrons and collisionless ions, predicts approximately a density drop at the MP entrance $n_{M P}(y, x)=n(y, x) / 2$ [29]. In that case, one obtains $\sigma=1 /\left(2 \pi N_{\text {turns }}\right)$. However, in general, experimental measurements show strong deviation from the theoretical estimate (see, e.g., Refs. $[29,30])$. Therefore, the value of $n_{M P}$ is usually not well known, leading to large uncertainties on $\sigma$.

To further simplify the problem, we use the isothermal plasma approximation, i.e. $T_{e}=1$, and we expand $\exp (\Lambda-\phi) \simeq 1+\Lambda-\phi$ and $n \ln (n) \simeq n-1$ assuming $\phi$ close to $\Lambda$ and $n$ 
close to 1, respectively. Equations (20)-(22) are thus rewritten as

$$
\begin{aligned}
\partial_{t} \theta= & -R_{0}\left\{\phi^{\prime}, \theta\right\}+2\left(\partial_{y} \theta-\partial_{y} \phi^{\prime}\right)-\sigma\left(1-\phi^{\prime}\right)+\mathcal{D}_{n}(\theta) \\
& +D_{n}\left[\left(\partial_{x} \theta\right)^{2}+\left(\partial_{y} \theta\right)^{2}\right]+S_{n}(1-\theta), \\
\partial_{t} \omega=- & R_{0}\left\{\phi^{\prime}, \omega\right\}+2 \partial_{y} \theta+\sigma \phi^{\prime}+\mathcal{D}_{\omega}(\omega),
\end{aligned}
$$

where $\omega=\nabla_{\perp}^{2} \phi^{\prime}, \phi^{\prime}=\phi-\Lambda$, and $\theta=\log (n)$. The model in Eqs. (23)-(24) is an ideal test bed for the application of the WRM discussed in Sec. II, since it contains both first and second order derivatives and nonlinear convective terms.

We note that the scalar fields $\theta, \omega$, and $\phi^{\prime}$ depend on time, $t \in\left[t_{0}, t_{1}\right]$, on the spatial coordinates $x \in\left[x_{0}, x_{1}\right]$ and $y \in\left[y_{0}, y_{1}\right]$, and on the parameter $\sigma$, which is estimated with a large uncertainty. Our goal is to estimate the impact of variations of $\sigma$ in an interval $\left[\sigma_{0}, \sigma_{1}\right]$ on the solution of Eqs. (23)-(24).

\section{NONLINEAR SIMULATIONS}

To illustrate an application of the Chebyshev spectral method illustrated in Sec. II to the study of uncertainty propagation, we present here the simulation of plasma turbulence in an SMT configuration, carried out considering the model discussed in Sec. III. First, we implement the model in a numerical code using the WRM approach and the decomposition in Chebyshev polynomials described in Sec. II. We then perform simulations of plasma turbulence in an SMT configuration by using the developed code. Finally, we investigate the impact of $\sigma$, the input parameter that characterizes the plasma losses at the vessel, on the time-averaged plasma profiles and on its fluctuations.

\section{A. Numerical implementation}

In order to investigate the dependence of $n, \phi^{\prime}$ and $\omega$ on $\sigma$, we developed a simulation code that solves Eqs. (23)-(24), together with $\omega=\nabla_{\perp}^{2} \phi^{\prime}$, implementing the WRM with Chebyshev 
decomposition described in Sec. II. More precisely, we write

$$
\begin{aligned}
\theta(t, x, y ; \sigma) & \simeq \sum_{k=0}^{K}{ }^{\prime} \sum_{l=0}^{L}, \sum_{m=0}^{M}, \sum_{n=0}^{N} ' a_{k l m n}^{\theta} T_{k}(\tau) T_{l}(\chi) T_{m}(v) T_{n}(\mu), \\
\omega(t, x, y ; \sigma) & \simeq \sum_{k=0}^{K}{ }^{\prime} \sum_{l=0}^{L}, \sum_{m=0}^{M}, \sum_{n=0}^{N} ' a_{k l m n}^{\omega} T_{k}(\tau) T_{l}(\chi) T_{m}(v) T_{n}(\mu), \\
\phi^{\prime}(t, x, y ; \sigma) & \simeq \sum_{k=0}^{K}{ }^{\prime} \sum_{l=0}^{L}, \sum_{m=0}^{M}, \sum_{n=0}^{N}{ }^{\prime} a_{k l m n}^{\phi} T_{k}(\tau) T_{l}(\chi) T_{m}(v) T_{n}(\mu),
\end{aligned}
$$

where $K, L, M$, and $N$ are the highest-order Chebyshev polynomials used for the decomposition along the temporal, radial, vertical, and parameter coordinates, respectively, and

$$
\tau=\frac{t-A_{t}}{B_{t}}, \chi=\frac{x-A_{x}}{B_{x}}, v=\frac{y-A_{y}}{B_{y}}, \mu=\frac{\sigma-A_{\sigma}}{B_{\sigma}},
$$

with $A_{t}=\left(t_{1}+t_{0}\right) / 2$ and $B_{t}=\left(t_{1}-t_{0}\right) / 2$ (similar definitions apply to the other quantities). Following the procedure described in Appendix A, we write the operators $\{\phi, A\}, \nabla_{\perp}^{2} A, \partial_{x} A$, $\partial_{y} A$, as well as the terms $\sigma\left(1-\phi^{\prime}\right), \sigma \phi^{\prime}$, and $S_{n}(1-\theta)$, in the Chebyshev spectral domain obtaining, according to Eq. (15), a set of $3 K(L-1)(M-1)(N+1)$ algebraic nonlinear equations for the coefficients $a_{k l m n}^{\theta}, a_{k l m n}^{\omega}$, and $a_{k l m n}^{\phi}$, with $0<k \leq K, 0 \leq l \leq L-2$, $0 \leq m \leq M-2,0 \leq n \leq N$. The $k=0$ coefficients are evaluated by applying the initial condition as described in Appendix B, while the $l=L-1, l=L, m=M-1$, and $m=M$ coefficients are computed by applying the boundary conditions as discussed in Appendix C. To solve numerically the resulting system of equations, we write the equation $\omega=\nabla_{\perp}^{2} \phi^{\prime}$ in the Chebyshev spectral domain as a linear system $\underline{\underline{A}} \mathbf{a}^{\phi}=\mathbf{a}^{\omega}$, with $\underline{\underline{A}}$ a square matrix with inverse $\underline{\underline{A}}^{-1}$, whereas $\mathbf{a}^{\omega}$ and $\mathbf{a}^{\phi}$ are vectors containing respectively the coefficients $a_{k l m n}^{\omega}$ and $a_{k l m n}^{\phi}$. This allows us to express $\mathbf{a}^{\phi}=\underline{\underline{A}}^{-1} \mathbf{a}^{\omega}$. Using the expression of $\mathbf{a}^{\phi}$ as a function of $\mathbf{a}^{\omega}$ in Eqs. (23)-(24), we obtain a set of $2(K+1)(L+1)(M+1)(N+1)$ algebraic nonlinear equations for the coefficients $a_{k l m n}^{\theta}$ and $a_{k l m n}^{\omega}$ in the form

$$
\left[\begin{array}{l}
\mathbf{a}^{\theta} \\
\mathbf{a}^{\omega}
\end{array}\right]=\mathbf{f}\left(\left[\begin{array}{l}
\mathbf{a}^{\theta} \\
\mathbf{a}^{\omega}
\end{array}\right]\right),
$$

with $\mathbf{a}^{\theta}$ a vector containing the coefficients $a_{k l m n}^{\theta}$ and $\mathbf{f}$ a vector function reflecting Eqs. (23)(24) in Chebyshev spectral domain.

The nonlinear system in Eq. (29) is implemented in a numerical code written in Fortran90 and interfaced with the MATLAB environment [31] and it is solved with the MATLAB fsolve nonlinear system solver using a trust-region algorithm. To facilitate the solver convergence, 
we separate the time coordinate in sub-domains as described in Sec. II (the results we show consider $\Delta t=\Delta t^{i}=0.2$ and we verified numerically that they are converged with respect to $\Delta t)$.

\section{B. Simulation results}

For our simulations we consider a spatial domain extending radially from $x_{0}=0$ to $x_{1}=80$ and vertically from $y_{0}=0$ to $y_{1}=80$. Moreover, we consider a parameter $\sigma$ varying between $\sigma_{0}=0.05$ and $\sigma_{1}=0.1$. At $x_{0}$ and $x_{1}$ we impose Dirichlet boundary conditions for $\phi^{\prime}$ and $\omega$, while we use Neumann boundary conditions for $\theta$. Because of the assumption $k_{z}=0$, we impose periodic boundary conditions at $y_{0}$ and $y_{1}$. Moreover, we use a source term of the form $S_{n}(x) \propto \exp \left[-\left(x-x_{S}\right)^{2} / 16\right]$, with $x_{S}=20$, and we set $D_{n}=D_{\omega}=5$ and $R_{0}=200$. We note that similar parameters were used for TORPEX simulations in Ref. [10]. Simulations are initialized from random noise and, after a transient phase, a quasi-stationary state is reached, in which the plasma, injected in the system by the plasma source, is lost because of parallel losses, modeled by $\sigma$, and losses at the inner and outer boundaries. We note that all the results presented in the following of the present paper are obtained during a time interval of approximately 40 time units during the quasi-stationary state.

Figure 1 shows typical snapshots of the plasma density for $\sigma=0.05,0.075,0.1$, resulting

from a simulation performed with $(K, L, M, N)=(1,22,22,3)$. The turbulent character of the plasma dynamics observed in previous finite difference simulations (see, e.g., Ref. [32]) is retrieved also by our WRM simulation, with eddies extending radially outward from the source location and that detach from it, creating blobs that propagate towards the low-field side part of the domain. We remark that the WRM approach makes it possible to simulate the TORPEX plasma dynamics for any value of $\sigma$ between 0.05 and 0.1 solving once Eq. (29).

\section{Uncertainty propagation}

Numerical simulations of plasma turbulence in basic plasma physics experiments, as well as in the tokamak SOL, are often employed to evaluate time-averaged quantities (e.g., the time-averaged pressure gradient length $L_{p}=-\left\langle p_{e}\right\rangle_{t} / \nabla\left\langle p_{e}\right\rangle_{t}$, with $\left\langle p_{e}\right\rangle_{t}$ the time-averaged plasma pressure [33]) or the fluctuation level (see, e.g., Ref. [34]). Therefore, as an example 

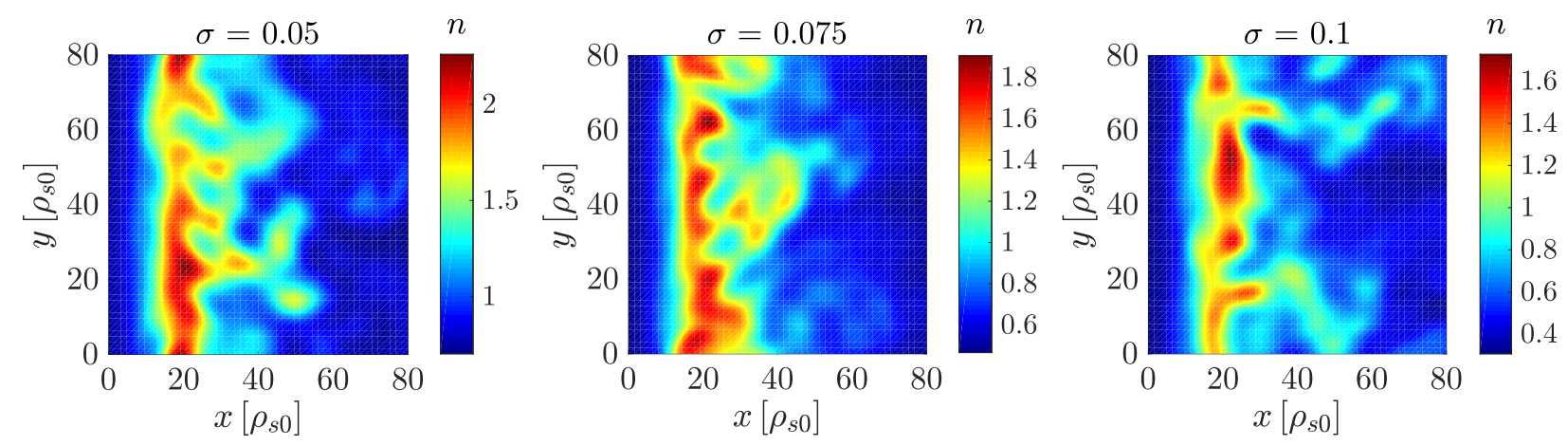

FIG. 1: Typical snapshots of plasma density for $\sigma=0.05,0.075,0.1$ (left, middle, and right panels, respectively), obtained by evaluating $\theta$ according to Eq. (25) with $(K, L, M, N)=(1,22,22,3)$.

of uncertainty propagation study, in the following we focus on $\eta(x ; \sigma)=\langle n(t, x, y ; \sigma)\rangle_{y, t}$ and $\delta \eta(x ; \sigma)=\langle\delta n(x, y ; \sigma)\rangle_{y} /\langle n(t, x, y ; \sigma)\rangle_{y, t}$, where $\langle-\rangle_{y}$ denotes vertical-averaging, $\langle-\rangle_{y, t}$ denotes vertical- and time-averaging, and $\delta n$ is the root-mean-square (RMS) value of density fluctuations. The density time-averages and the density fluctuation RMS values presented in the rest of the present section are computed numerically, evaluating $n(t, x, y ; \sigma)=\exp [\theta(t, x, y ; \sigma)]$ on a numerical grid characterized by $N_{x}=256, N_{y}=128$, and $\Delta t=0.05$, where $\theta(t, x, y ; \sigma)$ is the expansion in Eq. (25) that satisfies Eq. (29), and $N_{x}$ and $N_{y}$ are the number of points in the radial and vertical directions (we verified that the numerical results are converged in $N_{x}, N_{y}$, and $\Delta t$ ).

In Fig. 2 we present the profiles of $\eta(x ; \sigma)$ for $\sigma=0.5,0.075,0.1$ (left, middle, and right panels, respectively) obtained from three WRM simulations with $(K, L, M, N)=(1,12,9,1)$, $(2,14,11,2),(3,16,13,3)$ (blue, red, and yellow lines, respectively). The profiles are compared to the results of converged simulations performed with a finite difference code [10] that solves Eqs. (23)-(24) (black lines). We observe that the results obtained with the WRM are consistent with simulations carried out with the finite difference approach. The differences are probably due to the relatively small number of spectral terms used in the decomposition of $\theta, \phi^{\prime}$, and $\omega$.

To investigate quantitatively the impact of $\sigma$ on the simulation results, we consider (i) the time-averaged density gradient length $L_{n}(\sigma)=-\eta / \partial_{x} \eta$, (ii) the averaged density value, $\eta(x ; \sigma)$, evaluated at $x=40$ and $x=60$, and (iii) the density fluctuation level, $\delta \eta(x ; \sigma)$, evaluated at $x=40$ and $x=60$. Note that we assume that the averaged density profile decreases exponentially for $x>x_{S}$ to compute $L_{n}$, i.e. $\eta(x ; \sigma) \propto \exp \left[-\left(x-x_{S}\right) / L_{n}(\sigma)\right]$, and 

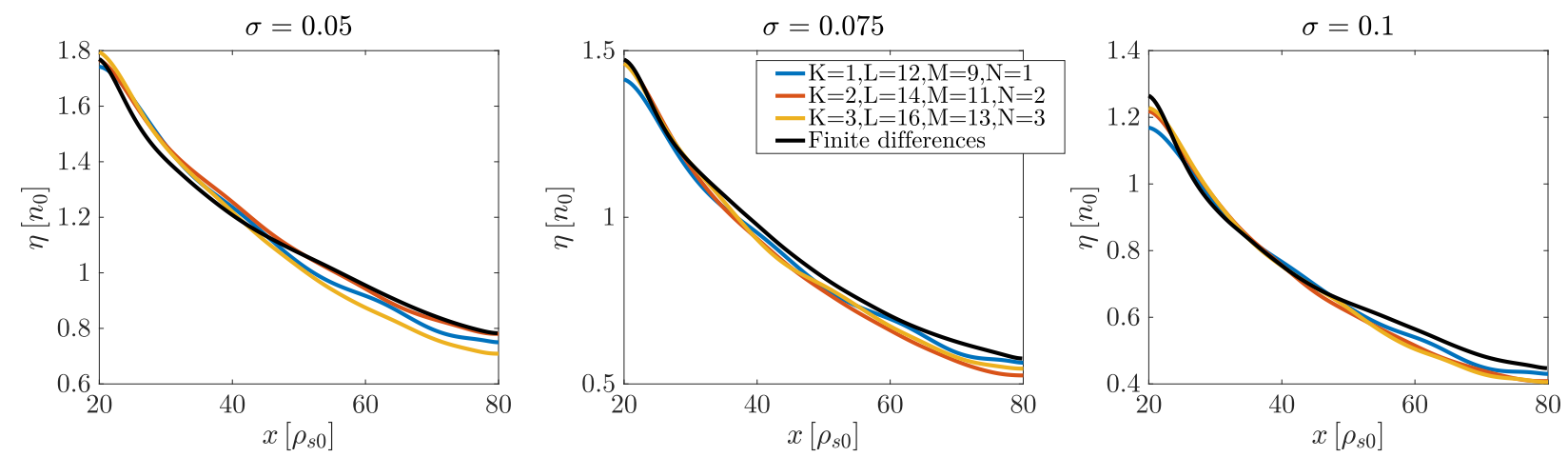

FIG. 2: Radial profiles of $\eta(x ; \sigma)$ for $\sigma=0.5,0.075,0.1$ (left, middle, and right panels, respectively). The simulation results are obtained by using a Chebyshev decomposition with $(K, L, M, N)=$ $(1,12,9,1),(2,14,11,2),(3,16,13,3)$ (blue, red, and yellow lines, respectively), and by using the finite difference code [10] that solves Eqs. (23)-(24) (black lines).

we evaluate $L_{n}(\sigma)$ as

$$
L_{n}(\sigma)=\left[x_{1 / 2}(\sigma)-x_{S}\right] / \ln (2),
$$

with $x_{1 / 2}(\sigma)$ satisfying $\eta\left(x_{1 / 2} ; \sigma\right)=\eta\left(x_{S} ; \sigma\right) / 2$.

To study the impact of $\sigma$ on $L_{n}, \eta$, and $\delta \eta$, we assume that $\sigma$ is characterized by a probability density function

$$
f_{\sigma}(\sigma) \propto \exp \left[-\left(\sigma-A_{\sigma}\right)^{2} / \mathrm{SD}(\sigma)^{2}\right]
$$

with $A_{\sigma}=\left(\sigma_{1}+\sigma_{0}\right) / 2=0.75$ and $\mathrm{SD}(\sigma)=0.005$ the standard deviation, and we randomly generate $R \simeq 1000$ samples $\sigma^{i}, i=1,2, \ldots, R$, distributed according to $f_{\sigma}$. Then, for each element $\sigma^{i}$ we compute the corresponding $L_{n}\left(\sigma^{i}\right)$. The results thus obtained are presented in Fig. 3 for $(K, L, M, N)=(1,12,9,1),(2,14,11,2),(3,16,13,3)$ (left, middle, and right panels, respectively). We observe that $L_{n}$ does not depend linearly on $\sigma$, since the three $L_{n}$ distributions do not exhibit the same Gaussian distribution as $\sigma$. We remark that the distributions of $L_{n}$ display a dependence on the number of spectral terms used in the simulation, pointing out that the numerical results are not fully converged at the relatively low $(K, L, M, N)$ used.

In the first row of Table I we present the values of $L_{n}$ averaged over the three distributions shown in Fig. 3. The uncertainties on the averaged $L_{n}$ values are evaluated as the standard deviation of each distribution, i.e. $\epsilon_{L_{n}}=\operatorname{SD}\left(L_{n}\right)$. We observe that the averaged $L_{n}$ values display a non negligible dependence on $(K, L, M, N)$. On the other hand, the uncertainties 

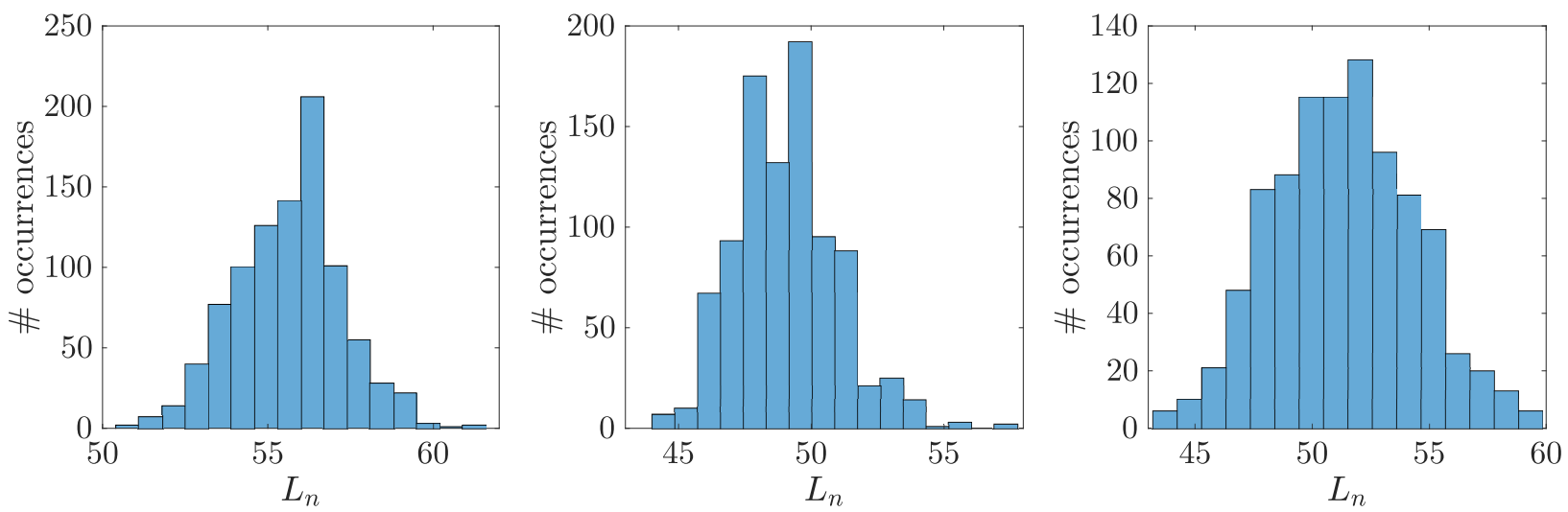

FIG. 3: Distributions of $L_{n}\left(\sigma^{i}\right)$, evaluated for approximately one thousand $\sigma^{i}$ s randomly generated according to Eq. (31), computed according to Eq. (30) and displayed for $(K, L, M, N)=$ $(1,12,9,1),(2,14,11,2),(3,16,13,3)$ (left, middle, and right panels, respectively).

TABLE I: Averaged values of the distributions shown in Figs. 3, 4 , and 5 for $(K, L, M, N)=$ $(1,12,9,1),(2,14,11,2),(3,16,13,3)$ (first, second, and third columns, respectively), with the uncertainties evaluated as the standard deviation of each distribution, and simulation results obtained with a finite difference code for $\sigma=\bar{\sigma}$ and $\sigma=\bar{\sigma} \pm \epsilon_{\sigma}$ (last three columns).

\begin{tabular}{lcccccccc}
\hline & \multicolumn{3}{c}{ Spectral code } & \multicolumn{3}{c}{ Finite difference code } \\
\cline { 2 - 4 } & $(1,12,9,1)$ & $(2,14,11,2)$ & $(3,16,13,3)$ & & $\bar{\sigma}-\epsilon_{\sigma}$ & $\bar{\sigma}$ & $\bar{\sigma}+\epsilon_{\sigma}$ \\
\hline$L_{n}$ & $56 \pm 2$ & $49 \pm 2$ & $51 \pm 3$ & & 52.7 & 53.2 & 53.2 \\
$\eta$ at $x=40$ & $0.96 \pm 0.05$ & $0.94 \pm 0.05$ & $0.94 \pm 0.05$ & 1.01 & 0.97 & 0.90 \\
$\eta$ at $x=60$ & $0.70 \pm 0.04$ & $0.66 \pm 0.04$ & $0.68 \pm 0.05$ & & 0.74 & 0.70 & 0.67 \\
$\delta \eta$ at $x=40$ & $0.15 \pm 0.01$ & $0.24 \pm 0.01$ & $0.20 \pm 0.02$ & & 0.18 & 0.18 & 0.19 \\
$\delta \eta$ at $x=60$ & $0.11 \pm 0.01$ & $0.19 \pm 0.01$ & $0.15 \pm 0.01$ & 0.14 & 0.14 & 0.15 \\
\hline
\end{tabular}

on $L_{n}$ are similar for different numbers of spectral terms. Since the difference between these uncertainties is small, we conclude that it is possible to obtain a rough estimate of the spread of $L_{n}$, due to variations of $\sigma$, by considering a small number of spectral terms, which is exactly the target of our methodology. This is particularly remarkable, since the simulation performed with $(K, L, M, N)=(1,12,9,1)$ is less demanding, in terms of computational resources, by approximately a factor 350 with respect to the simulation carried out with $(K, L, M, N)=(3,16,13,3)$. 

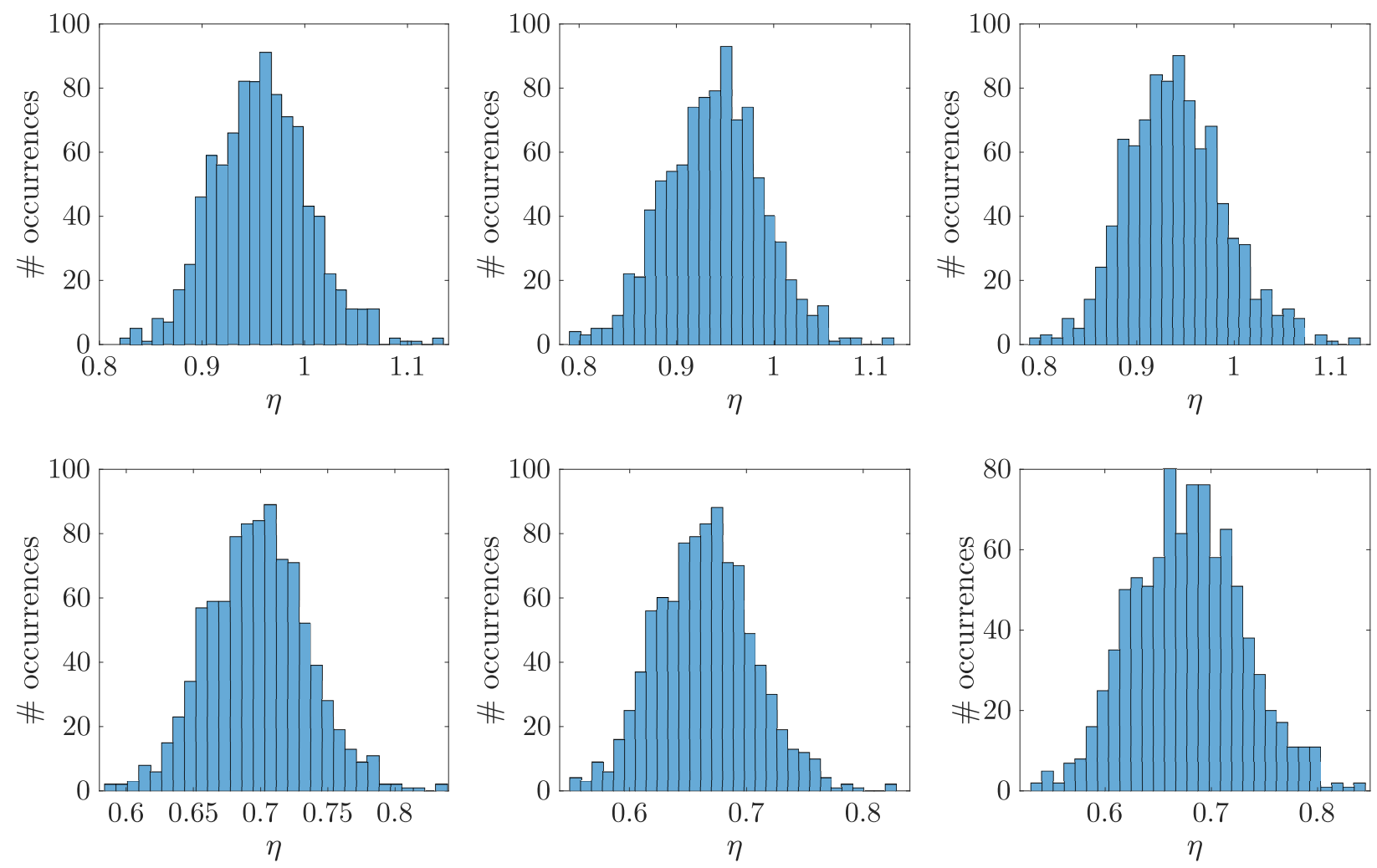

FIG. 4: Distributions of $\eta\left(x ; \sigma^{i}\right)$, evaluated at $x=40$ (first row) and $x=60$ (second row) for approximately one thousand $\sigma^{i}$ s randomly generated according to Eq. (31) and displayed for $(K, L, M, N)=(1,12,9,1),(2,14,11,2),(3,16,13,3)$ (left, middle, and right columns, respectively).

It is interesting to compare the results obtained with the spectral method with a standard sensitivity study performed with a finite difference approach. This is performed according to the standard practice. We assume that the uncertainty affecting $\sigma$ corresponds to the half width half maximum $(\mathrm{HFHM})$ of the distribution $f_{\sigma}$, i.e. we assume $\epsilon_{\sigma}=\sqrt{2 \ln 2} \mathrm{SD}(\sigma) \simeq$ 0.006. Then, we perform three finite difference simulations, one for $\sigma=\bar{\sigma}=0.075$, one for $\sigma=\bar{\sigma}-\epsilon_{\sigma}=0.069$, and one for $\sigma=\bar{\sigma}+\epsilon_{\sigma}=0.081$. The values of $L_{n}$ obtained from these three simulations are shown in the fourth, fifth, and sixth columns of the first row of Table I. We observe that the averaged values of $L_{n}$ obtained with the spectral code are similar to the ones obtained from a finite difference simulation for $\sigma=\bar{\sigma}$ (the difference is within the numerical uncertainty due to the number of spectral terms used in the WRM simulations). On the other hand, the uncertainty on $L_{n}$ estimated as the difference between the three finite difference simulations is below 0.5, while the uncertainties evaluated with the spectral code are at least a factor of four larger. This shows that, in this particular 

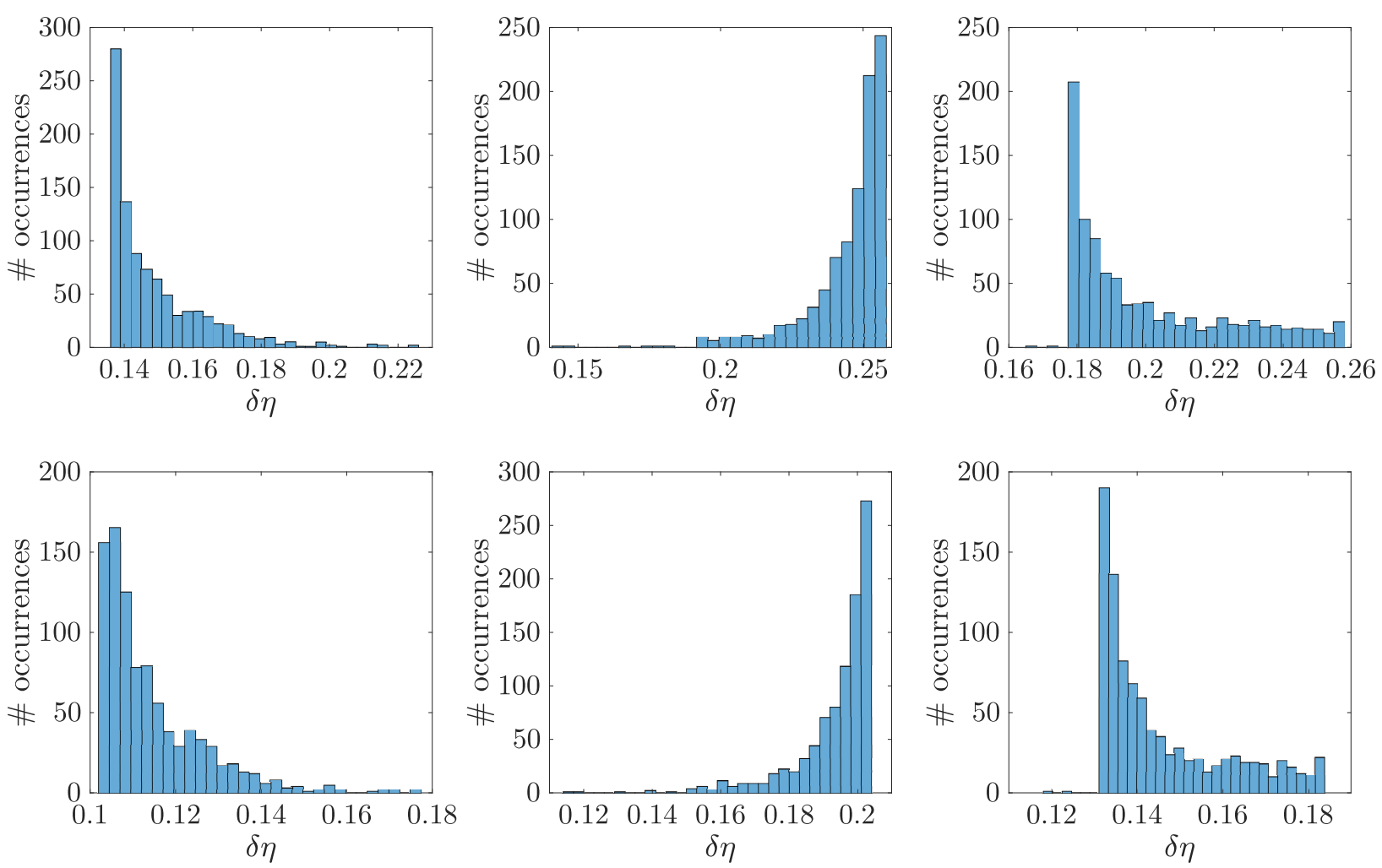

FIG. 5: Distributions of $\delta \eta\left(x ; \sigma^{i}\right)$, evaluated at $x=40$ (first row) and $x=60$ (second row) for approximately one thousand $\sigma^{i}$ s randomly generated according to Eq. (31) and displayed for $(K, L, M, N)=(1,12,9,1),(2,14,11,2),(3,16,13,3)$ (left, middle, and right columns, respectively)

situation, a standard sensitivity scan significantly underestimates the uncertainty on $L_{n}$ due to uncertainties on $\sigma$.

A similar study to the one performed for $L_{n}$ is also carried out for local values of $\eta$. In Fig. 4 we present the distributions of $\eta$ obtained at $x=40$ (first row) and $x=60$ (second row) with the spectral code for $(K, L, M, N)=(1,12,9,1),(2,14,11,2),(3,16,13,3)$ (left, middle, and right columns, respectively). We observe that the distributions slightly depend on the number of spectral terms used in the simulations and that $\eta$ does not depend linearly on $\sigma$. The averaged values of the six distributions shown in Fig. 4 are illustrated in the second and third rows of Table I together with the corresponding standard deviations, the latter denoted as uncertainties on the averaged values. We observe that the averaged values of $\eta$ slightly depend on the number of spectral terms used for the simulations and that the values of the standard deviation of the six distributions are close to each other. For comparison, in the last three columns of Table I, second and third rows, we display $\eta$ obtained with the 
finite difference code for $\sigma=\bar{\sigma}$ and $\sigma=\bar{\sigma} \pm \epsilon_{\sigma}$. In this case, the difference in $\eta$ between the three finite difference simulations used to estimate the uncertainty in the simulation results is comparable to the uncertainties estimated with the spectral code.

Finally, in Fig. 5 we display the distributions of $\delta \eta$ obtained at $x=40$ (first row) and $x=60$ (second row) for $(K, L, M, N)=(1,12,9,1),(2,14,11,2),(3,16,13,3)$ (left, middle, and right columns, respectively) with the spectral code. We note that the $\delta \eta$ distributions are extremely different from a Gaussian distribution and they depend on the number of spectral terms used in the simulations. However, their averaged values (shown in the last two rows of Table I, first three columns), as well as their standard deviations (shown in Table I as uncertainty on $\delta \eta$ ), are similar. Comparing the spectral results with the numerical results obtained with the finite difference approach (last two rows, last three columns of Table I), we notice that the two methodologies provide similar results, both for the averages and for the estimated uncertainties.

In summary, the averaged values of $L_{n}, \eta$, and $\delta \eta$ obtained with the spectral code are comparable to the results obtained with the finite difference code for $\sigma=\bar{\sigma}=0.075$. On

the other hand, the spectral code provides, with a single reduced-cost simulation, a more reliable estimate of the uncertainty affecting these quantities due to the $\sigma$ uncertainty.

\section{CONCLUSIONS}

In the present paper we discuss a rigorous methodology to assess the uncertainty affecting a simulation result due to the propagation of input parameter uncertainties. More precisely, in order to study the impact of input parameter variations on the results of a plasma turbulence model, we propose to use a WRM with decomposition in Chebyshev polynomials. This choice is motivated by the minimax property, which ensures that the best approximation of the model solution at the chosen order and under the $L_{\infty}$ norm is given by the Chebyshev decomposition. By applying the WRM, a system of nonlinear algebraic equations is derived for the coefficients of the Chebyshev expansion. The solution of these equations directly provides information on the dependence of the simulation results on the input parameters [11]. We apply the proposed methodology to a two-dimensional drift-reduced Braginksii model used to investigate the plasma dynamics in basic plasma physics experiments and in the tokamak SOL. These equations are decomposed in the Chebyshev spectral domain and the 
resulting system of equations is implemented in a numerical code. The plasma turbulent dynamics is retrieved by our simulations and an explicit dependence of the profiles on the parameter describing the parallel losses is obtained.

Assuming that the input parameter under consideration is distributed according to a Gaussian probability distribution function, we compute the standard deviation that characterizes the corresponding (in principle, non-Gaussian) distribution of time-averaged density gradient lengths, and time-averaged and fluctuation density values. We find that a reasonable value of the spread of $L_{n}, \eta$, and $\delta \eta$, due to the uncertainty affecting the input parameter that describes the parallel losses, can be obtained using a small number of Chebyshev polynomials, i.e. by carrying out reduced-cost simulations. Our results are compared to the outcome of a standard sensitivity study, pointing out that the spectral method employed is more reliable in estimating the uncertainties on the simulation results due to uncertainties on input parameters than a finite difference approach. To our knowledge, this is the first time that a fully spectral approach is used to successfully simulate plasma turbulence and study uncertainty propagation.

\section{Acknowledgments}

The simulations presented herein were carried out in part at the Swiss National Supercomputing Center (CSCS) under Project ID s718; and in part on the CINECA Marconi supercomputer within the framework of the GBSSOL project. This work has been carried out within the framework of the EUROfusion Consortium and has received funding from the Fond National Suisse de la Recherche scientifique and from the Euratom research and training programme 2014-2018 under grant agreement No 633053. The views and opinions expressed herein do not necessarily reflect those of the European Commission.

\section{APPENDIX A: OPERATORS IN THE CHEBYSHEV SPECTRAL DOMAIN}

In order to relate the coefficients $a_{k l m}$ in Eq. (4), used to represent $u$ in the Chebyshev spectral domain, with the coefficients $c_{k l m}$ in Eq. (16), one has to express differential and nonlinear operators in Chebyshev space. This is performed by exploiting some useful properties of Chebyshev polynomials, as summarized in the rest of this appendix (see Ref. [20] 
for a more detailed discussion). For simplicity, we consider functions of one variable $x$. The generalization to multiple variables does not present any conceptual difficulty.

\section{Differential operators}

First-order spatial derivatives of a function can be easily obtained by exploiting the property

$$
\frac{d T_{n}}{d x}(\chi)=\frac{2 n}{B_{x}} \sum_{\substack{l \geq 0 \\ n-l \text { odd }}}^{n-1} T_{l}(\chi)
$$

Therefore, as detailed in Ref. [11], the first-order derivative of a function $f(x)=\sum_{l=0}^{L}{ }^{\prime} a_{l} T_{l}(\chi)$ is computed as

$$
\frac{d f}{d x}(x)=\frac{d}{d x} \sum_{l=0}^{L}{ }^{\prime} a_{l} T_{l}(\chi)=\frac{2}{B_{x}} \sum_{l=0}^{L-1}, \sum_{\substack{\lambda=l+1 \\ \lambda-l \text { odd }}}^{L} \lambda a_{\lambda} T_{l}(\chi) .
$$

Similarly, second order derivatives of $f(x)$ are expressed as [11]

$$
\frac{d^{2} f}{d x^{2}}(x)=\frac{d^{2}}{d x^{2}} \sum_{l=0}^{L}{ }^{\prime} a_{l} T_{l}(\chi)=\frac{1}{B_{x}^{2}} \sum_{l=0}^{L-2}{ }_{\substack{\lambda=l+2 \\ \lambda-l \text { even }}}^{L} \lambda\left(\lambda^{2}-l^{2}\right) a_{\lambda} T_{l}(\chi) .
$$

Higher order derivatives are obtained iterating Eq. (A1). We remark that, when differentiating with respect to $x$ a function $f(x)$ represented in terms of Chebyshev polynomials of order $L$, the function $d f / d x$ only includes polynomials up to order $L-1$.

\section{Nonlinear terms}

Linear operators involving the addition or subtraction of two functions are easily handled in the Chebyshev spectral domain. On the other hand, care must be taken in computing nonlinear operators related, for example, to the multiplication of two functions.

Focusing on the product operator, that is the basis of all nonlinear operators, and exploiting the fact that Chebyshev polynomials satisfy

$$
T_{m}(x) T_{n}(x)=\frac{1}{2}\left[T_{m+n}(x)+T_{|m-n|}(x)\right],
$$


it is possible to write the product $h(x)=f(x) g(x)$ between the two functions $f(x)=$ $\sum_{l=0}^{L}{ }^{\prime} a_{l} T_{l}(\chi)$ and $g(x)=\sum_{l=0}^{L}{ }^{\prime} b_{l} T_{l}(\chi)$ as

$$
\begin{aligned}
h(x) & =\sum_{l=0}^{L}{ }^{\prime} \sum_{i=0}^{L}, \frac{a_{l} b_{i}}{2}\left[T_{l+i}(\chi)+T_{|l-i|}(\chi)\right] \\
& =\sum_{l=0}^{L},\left[\sum_{i=l}^{L+l} \frac{a_{l} b_{i-l}}{2} T_{i}(\chi)+\sum_{i=0}^{l-1} \frac{a_{l} b_{l-1}}{2} T_{i}(\chi)+\sum_{i=1}^{L-l} \frac{a_{l} b_{i+l}}{2} T_{i}(\chi)\right] .
\end{aligned}
$$

We now approximate

$$
h(x) \simeq \sum_{l=0}^{L}{ }^{\prime} c_{l} T_{l}(\chi)
$$

i.e. we truncate the expansion of $h(x)$ at order $L$ (because of the minimax property, this truncated series is the most accurate polynomial representation of $h(x)$ to order $L$ ). To express the coefficients $c_{l}$ in terms of $a_{l}$ and $b_{l}$, we impose

$$
\sum_{l=0}^{\infty}{ }^{\prime} c_{l} T_{l}(\chi)=\sum_{l=0}^{L},\left[\sum_{i=l}^{L+l} \frac{a_{l} b_{i-l}}{2} T_{i}(\chi)+\sum_{i=0}^{l-1} \frac{a_{l} b_{l-1}}{2} T_{i}(\chi)+\sum_{i=1}^{L-l} \frac{a_{l} b_{i+l}}{2} T_{i}(\chi)\right]
$$

and we multiply both sides of Eq. (A7) by $T_{p}(\chi) / \sqrt{1-\chi^{2}}$, with $0 \leq p \leq L$. Then, by applying Eq. (2), we obtain

$$
c_{l}=\sum_{i=0}^{l} \frac{a_{i} b_{l-i}}{2}+\sum_{i=1}^{L-l} \frac{a_{i} b_{i+l}+a_{i+l} b_{i}}{2}
$$

with $0 \leq l \leq L$.

\section{APPENDIX B: INITIAL CONDITION}

The coefficients $b_{l m}$ in Eq. (13), used to express the initial condition in Chebyshev space, are given by

$$
b_{l m}=\frac{1}{B_{x} B_{p}}\left(\frac{2}{\pi}\right)^{2} \int_{x_{0}}^{x_{1}} \int_{p_{0}}^{p_{1}} u_{0}(x ; p) \frac{T_{l}(\chi)}{\sqrt{1-\chi^{2}}} \frac{T_{m}(\sigma)}{\sqrt{1-\sigma^{2}}} d x d p .
$$

Since, in general, the integrals in Eq. (B1) cannot be computed analytically, we introduce an approximation of $b_{l m}$ that can be easily evaluated. In fact [11],

$$
b_{l m} \simeq \frac{2}{L+1} \frac{2}{M+1} \sum_{r=1}^{L+1} \sum_{s=1}^{M+1} u\left(t_{0}, x_{r} ; p_{s}\right) T_{l}\left(\chi_{r}\right) T_{m}\left(\sigma_{s}\right)
$$

where

$$
\chi_{r}=\cos \left[\frac{\pi}{L+1}\left(r-\frac{1}{2}\right)\right], \sigma_{s}=\cos \left[\frac{\pi}{M+1}\left(s-\frac{1}{2}\right)\right],
$$

$x_{r}=B_{x} \chi_{r}+A_{x}$, and $p_{s}=B_{p} \sigma_{s}+A_{p}$. The expression in Eq. (B2) approximates $b_{l m}$ in the limit $L, M \rightarrow \infty$ and it is easily generalized to continuous functions with more variables. 


\section{APPENDIX C: BOUNDARY CONDITIONS}

In this appendix we consider how to set the boundary conditions in the Chebyshev spectral domain. To simplify the discussion, we focus on differential equations where the operator $D_{p}$ involves second order derivatives of $u$ with respect to $x$. The generalization to higher order derivatives does not present any conceptual difficulty. Moreover, we consider that the same kind of boundary conditions (Dirichlet, Neumann, and periodic boundary) is applied at the two boundaries of the spatial domain. Combinations of different kinds of boundary conditions are easily obtained following the procedure described hereafter.

\section{Dirichlet boundary conditions}

To apply Dirichlet boundary conditions we follow the procedure described in Ref. [11] and summarized here for completeness. Since the expansion of $d^{2} u / d x^{2}$ leads to polynomials up to order $L-2$, the boundary conditions are set by imposing the $L-1$ and $L$ coefficients of the Chebyshev expansion. Considering the two boundary conditions

$$
u\left(t, x_{0} ; p\right)=\alpha(t ; p), \quad u\left(t, x_{1} ; p\right)=\beta(t ; p)
$$

and approximating

$$
\begin{aligned}
& \alpha(t ; p) \simeq \sum_{k=0}^{K}{ }^{\prime} \sum_{m=0}^{M}{ }^{\prime} \alpha_{k m} T_{k}(\tau) T_{m}(\sigma), \\
& \beta(t ; p) \simeq \sum_{k=0}^{K}, \sum_{m=0}^{M}{ }^{\prime} \beta_{k m} T_{k}(\tau) T_{m}(\sigma),
\end{aligned}
$$

the orthogonality property of Chebyshev polynomials implies that, to satisfy Eq. (C1),

$$
\begin{aligned}
& \alpha_{k m}=\sum_{l=0}^{L}{ }^{\prime} a_{k l m} T_{l}(-1)=\sum_{l=0}^{L}{ }^{\prime} a_{k l m}(-1)^{l}, \\
& \beta_{k m}=\sum_{l=0}^{L}{ }^{\prime} a_{k l m} T_{l}(1)=\sum_{l=0}^{L}{ }^{\prime} a_{k l m},
\end{aligned}
$$

where we use $T_{l}(-1)=(-1)^{l}$ and $T_{l}(1)=1$. Taking the sum and the difference of these two expressions and rearranging we obtain

$$
\begin{aligned}
& a_{k, L-1, m}=\frac{\beta_{k m}-\alpha_{k m}}{2}-\sum_{\substack{l=1 \\
l \text { odd }}}^{L-3} a_{k, l, m}, \\
& a_{k L m}=\frac{\alpha_{k m}+\beta_{k m}}{2}-\sum_{\substack{l=0 \\
l \text { even }}}^{L-2} a_{k, l, m}
\end{aligned}
$$


if $L$ is even, whereas we have

$$
\begin{gathered}
a_{k, L-1, m}=\frac{\alpha_{k m}+\beta_{k m}}{2}-\sum_{\substack{l=0 \\
l \text { even }}}^{L-3} a_{k, l, m}, \\
a_{k L m}=\frac{\beta_{k m}-\alpha_{k m}}{2}-\sum_{\substack{l=1 \\
l \text { odd }}}^{L-2} a_{k, l, m}
\end{gathered}
$$

if $L$ is odd.

\section{Neumann boundary conditions}

Neumann boundary conditions can be imposed in a similar way. Let us consider two boundary conditions of the form

$$
\frac{d u}{d x}\left(t, x_{0} ; p\right)=\alpha(t ; p), \quad \frac{d u}{d x}\left(t, x_{1} ; p\right)=\beta(t ; p),
$$

with

$$
\begin{aligned}
& \alpha(t ; p) \simeq \sum_{k=0}^{K}{ }^{\prime} \sum_{m=0}^{M}{ }^{\prime} \alpha_{k m} T_{k}(\tau) T_{m}(\sigma), \\
& \beta(t ; p) \simeq \sum_{k=0}^{K}, \sum_{m=0}^{M}{ }^{\prime} \beta_{k m} T_{k}(\tau) T_{m}(\sigma) .
\end{aligned}
$$

Using again the orthogonality property of Chebyshev polynomials, we obtain

$$
\begin{aligned}
& \alpha_{k m}=\frac{2}{B_{x}} \sum_{l=0}^{L-1}, \sum_{\substack{\lambda=l+1 \\
\lambda-l \text { odd }}}^{L} \lambda a_{k \lambda m}(-1)^{l}, \\
& \beta_{k m}=\frac{2}{B_{x}} \sum_{l=0}^{L-1}, \sum_{\substack{\lambda=l+1 \\
\lambda-l \text { odd }}}^{L} \lambda a_{k \lambda m} .
\end{aligned}
$$

An explicit expression for the coefficients $a_{k, L-1, m}$ and $a_{k L m}$ is easily computed by rearranging Eq. (C8), that is

$$
\begin{aligned}
& a_{k, L-1, m}=B_{x} \frac{\alpha_{k m}+\beta_{k m}}{2(L-1)^{2}}-\sum_{\substack{l=1 \\
l \text { odd }}}^{L-3} \frac{l^{2}}{(L-1)^{2}} a_{k l m}, \\
& a_{k L m}=B_{x} \frac{\beta_{k m}-\alpha_{k m}}{2 L^{2}}-\sum_{\substack{l=2 \\
l \text { even }}}^{L-2} \frac{l^{2}}{L^{2}} a_{k l m}
\end{aligned}
$$


if $L$ is even, whereas we have

$$
\begin{aligned}
& a_{k, L-1, m}=B_{x} \frac{\beta_{k m}-\alpha_{k m}}{2(L-1)^{2}}-\sum_{\substack{l=2 \\
l \text { even }}}^{L-3} \frac{l^{2}}{(L-1)^{2}} a_{k l m}, \\
& a_{k L m}=B_{x} \frac{\alpha_{k m}+\beta_{k m}}{2 L^{2}}-\sum_{\substack{l=1 \\
l \text { odd }}}^{L-2} \frac{l^{2}}{L^{2}} a_{k l m}
\end{aligned}
$$

if $L$ is odd.

\section{Periodic boundary conditions}

In order to apply the periodic boundary conditions

$$
u\left(t, x_{0} ; p\right)=u\left(t, x_{1} ; p\right), \quad \frac{d u}{d x}\left(t, x_{0} ; p\right)=\frac{d u}{d x}\left(t, x_{1} ; p\right),
$$

we impose in the Chebyshev spectral domain

$$
\begin{aligned}
& \sum_{l=0}^{L} ' a_{k l m}(-1)^{l}=\sum_{l=0}^{L} ' a_{k l m}, \\
& \sum_{l=0}^{L-1} '(-1)^{l} \sum_{\substack{\lambda=l+1 \\
\lambda-l \text { odd }}}^{L} \lambda a_{k \lambda m}=\sum_{l=0}^{L-1}, \sum_{\substack{\lambda=l+1 \\
\lambda-l \text { odd }}}^{L} \lambda a_{k \lambda m} .
\end{aligned}
$$

These expressions are rewritten as

$$
\sum_{\substack{l=1 \\ l \text { odd }}}^{L} a_{k l m}=0, \sum_{\substack{l=2 \\ l \text { even }}}^{L} l^{2} a_{k l m}=0 .
$$

The coefficients $a_{k, L-1, m}$ and $a_{k L m}$ are thus given as

$$
a_{k, L-1, m}=-\sum_{\substack{l=1 \\ l \text { odd }}}^{L-3} a_{k l m}, a_{k L m}=-\sum_{\substack{l=2 \\ l \text { even }}}^{L-2} \frac{l^{2}}{L^{2}} a_{k l m}
$$

if $L$ is even, whereas we have

$$
a_{k, L-1, m}=-\sum_{\substack{l=2 \\ l \text { even }}}^{L-3} \frac{l^{2}}{(L-1)^{2}} a_{k l m}, a_{k L m}=-\sum_{\substack{l=1 \\ l \text { odd }}}^{L-2} a_{k l m}
$$

if $L$ is odd.

[1] P. W. Terry, M. Greenwald, J.-N. Leboeuf, G. R. McKee, D. R. Mikkelsen, W. M. Nevins, D. E. Newman, and D. P. Stotler. Validation in fusion research: Towards guidelines and best practices. Physics of Plasmas 15, 062503 (2008). 
[2] M. Greenwald. Verification and validation for magnetic fusion. Physics of Plasmas 17, 058101 (2010).

[3] C. Holland. Validation metrics for turbulent plasma transport. Physics of Plasmas 23, 060901 (2016)

[4] F. Riva, P. Ricci, F. D. Halpern, S. Jolliet, J. Loizu, and A. Mosetto. Verification methodology for plasma simulations and application to a scrape-off layer turbulence code. Physics of Plasmas 21, 062301 (2014).

[5] F. Riva, C. F. Beadle, and P. Ricci. A methodology for the rigorous verification of Particlein-Cell simulations. Physics of Plasmas 24, 055703 (2017).

[6] W. L. Oberkampf, T. G. Trucano, and C. Hirsch. Verification, validation, and predictive capability in computational engineering and physics. Applied Mechanics Reviews 57, 345 (2004).

[7] B. Peherstorfer, K. Willcox, and M. Gunzburger. Optimal Model Management for Multifidelity Monte Carlo Estimation. SIAM Journal on Scientific Computing 38, A3163-A3194 (2016).

[8] S. S. Isukapalli, A. Roy, and P. G. Georgopoulos. Stochastic Response Surface Methods (SRSMs) for Uncertainty Propagation: Application to Environmental and Biological Systems. Risk Analysis 18, 351-363 (1998).

[9] R. Myers, D. Montgomery, and C. Anderson-Cook. Response Surface Methodology: Process and Product Optimization Using Designed Experiments. Wiley Series in Probability and Statistics. Wiley (2016).

[10] P. Ricci, C. Theiler, a. Fasoli, I. Furno, K. Gustafson, D. Iraji, and J. Loizu. Methodology for turbulence code validation: Quantification of simulation-experiment agreement and application to the TORPEX experiment. Physics of Plasmas 18, 032109 (2011).

[11] J. Scheffel. A Spectral Method in Time for Initial-Value Problems. American Journal of Computational Mathematics 02, 173-193 (2012).

[12] B. N. Rogers and P. Ricci. Low-Frequency Turbulence in a Linear Magnetized Plasma. Physical Review Letters 104, 225002 (2010).

[13] P. Ricci, B. N. Rogers, and S. Brunner. High- and Low-Confinement Modes in Simple Magnetized Toroidal Plasmas. Physical Review Letters 100, 225002 (2008).

[14] P. Ricci and B. N. Rogers. Transport scaling in interchange-driven toroidal plasmas. Physics of Plasmas 16, 062303 (2009). 
[15] P. Ricci and B. N. Rogers. Turbulence Phase Space in Simple Magnetized Toroidal Plasmas. Physical Review Letters 104, 145001 (2010).

[16] V. Naulin, O. Garcia, A. Nielsen, and J. Rasmussen. Statistical properties of transport in plasma turbulence. Physics Letters A 321, 355-365 (2004).

[17] O. E. Garcia, V. Naulin, A. H. Nielsen, and J. J. Rasmussen. Computations of Intermittent Transport in Scrape-Off Layer Plasmas. Physical Review Letters 92, 165003 (2004).

[18] C. Canuto, M. Hussaini, A. Quarteroni, and J. Thomas A. Spectral Methods in Fluid Dynamics. Scientific Computation. Springer Berlin Heidelberg (2012).

[19] V. Naulin and A. H. Nielsen. Accuracy of Spectral and Finite Difference Schemes in 2D Advection Problems. SIAM Journal on Scientific Computing 25, 104-126 (2003).

[20] J. Mason and D. Handscomb. Chebyshev Polynomials. CRC Press (2002).

[21] J. Scheffel and A. A. Mirza. Time-Spectral Solution of Initial-Value Problems-Subdomain Approach. American Journal of Computational Mathematics 02, 72-81 (2012).

[22] A. Fasoli, B. Labit, M. McGrath, S. H. Müller, G. Plyushchev, M. Podestà, and F. M. Poli. Electrostatic turbulence and transport in a simple magnetized plasma. Physics of Plasmas 13, $055902(2006)$.

[23] I. Furno, F. Avino, A. Bovet, A. Diallo, A. Fasoli, K. Gustafson, D. Iraji, B. Labit, J. Loizu, S. H. Müller, G. Plyushchev, M. Podestà, F. M. Poli, P. Ricci, and C. Theiler. Plasma turbulence, suprathermal ion dynamics and code validation on the basic plasma physics device TORPEX. Journal of Plasma Physics 81, 345810301 (2015).

[24] K. W. Gentle and H. He. Texas Helimak. Plasma Science and Technology 10, 284-289 (2008).

[25] A. Zeiler, J. F. Drake, and B. Rogers. Nonlinear reduced Braginskii equations with ion thermal dynamics in toroidal plasma. Physics of Plasmas 4, 2134 (1997).

[26] D. A. Russell, D. A. D'Ippolito, and J. R. Myra. On relaxing the Boussinesq approximation in scrape-off layer turbulence (SOLT) model simulations. Bulletin of the American Physical Society, 54th Annual Meeting of the APS Division of Plasma Physics, vol 57, BP8.159. Providence, Rhode Island, USA (2012).

[27] G. Q. Yu, S. I. Krasheninnikov, and P. N. Guzdar. Two-dimensional modelling of blob dynamics in tokamak edge plasmas. Physics of Plasmas 13, 042508 (2006).

[28] K. Bodi, G. Ciraolo, P. Ghendrih, F. Schwander, E. Serre, and P. Tamain. Impact of the Boussinesq approximation in tokamak scrape-off layer turbulence. 38th EPS Conference on 
Plasma Physics, P1.121. Strasbourg, France (2011).

[29] P. Stangeby. The Plasma Boundary of Magnetic Fusion Devices. Series in Plasma Physics and Fluid Dynamics. Taylor \& Francis (2000).

[30] I. Furno, C. Theiler, V. Chabloz, A. Fasoli, and J. Loizu. Pre-sheath density drop induced by ion-neutral friction along plasma blobs and implications for blob velocities. Physics of Plasmas 21, 012305 (2014).

[31] MATLAB. version 9.1 (R2016b). The MathWorks Inc., Natick, Massachusetts (2016).

[32] P. Ricci, F. D. Halpern, S. Jolliet, J. Loizu, A. Mosetto, A. Fasoli, I. Furno, and C. Theiler. Simulation of plasma turbulence in scrape-off layer conditions: the GBS code, simulation results and code validation. Plasma Physics and Controlled Fusion 54, 124047 (2012).

[33] F. D. Halpern, P. Ricci, B. Labit, I. Furno, S. Jolliet, J. Loizu, A. Mosetto, G. Arnoux, J. P. Gunn, J. Horacek, M. Kočan, B. LaBombard, and C. Silva. Theory-based scaling of the SOL width in circular limited tokamak plasmas. Nuclear Fusion 53, 122001 (2013).

[34] P. Ricci, F. Riva, C. Theiler, A. Fasoli, I. Furno, F. D. Halpern, and J. Loizu. Approaching the investigation of plasma turbulence through a rigorous verification and validation procedure: A practical example. Physics of Plasmas 22, 055704 (2015). 